\title{
Evanescent Field Sensing in novel Flat Fiber
}

\author{
C. Holmes, F.R. Mahamd Adikan, A.S. Webb, J.C. Gates, C.B.E. Gawith, J.K. Sahu, P.G.R. Smith, D.N. Payne \\ Optoelectronics Research Centre, University of Southampton, UK \\ chh@orc.soton.ac.uk
}

\begin{abstract}
Recently developed novel 'flat fiber' substrate promises flexible, long-haul integrated optical devices. Here, we present the first demonstration of one such device; an evanescent field sensor, based upon direct UV written Bragg gratings.
\end{abstract}

(c) 2008 Optical Society of America

OCIS codes: (130.6010) Sensors; (130.2755) Glass waveguides; (060.2370) Fiber optics sensors.

\section{Introduction}

Integrated optical devices offer dense, multifunctional capability in a single robust package but are rarely considered compatible with the fields of remote or distributed sensing or compete in the long-haul with conventional 'one dimensional' fibers. Here we aim to change that by introducing a 'flat-fiber' process that combines the advantages of existing low-cost fiber drawing with the functionality of planar lightwave circuits in a novel hybrid format. Adapted from modified chemical vapour deposition (MCVD) fiber fabrication, our preforms are deposited and collapsed into a rectangular geometry (Figure 1 (a)) by applying a vacuum during the fibre drawing process [1], resulting in extended lengths of mechanically flexible flat-fiber material. Figure 1 (b) demonstrates the flexible glass planar substrate with lengths extending over a metre.

(a)

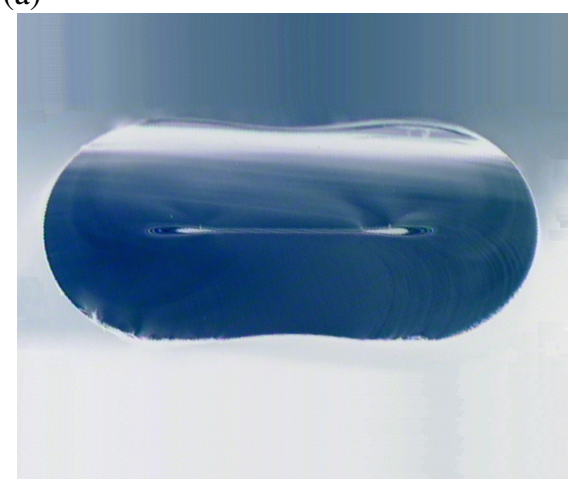

(b)

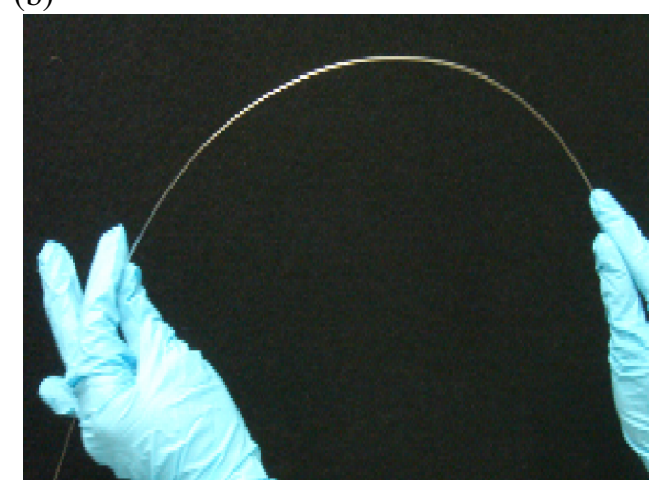

Figure 1: Extended lengths of the flexible glass planar substrate.

Doping the core layer of the flat fibre with germanium allows for photosensitivity. Due to the unique cross section profile of the substrate, and the thick cladding layer (in excess of $300 \mu \mathrm{m}$ ), the current technique that is most suited in defining waveguide structures is direct UV writing. The technique involves inscribing a pre-designed waveguide structure by translating a photosensitive sample beneath focussed dual UV beams [2], with refractive index increase of $10^{-4}$ to $10^{-3}$. We have previously demonstrated buried channel waveguides, and y-splitters using such approach and conducted a series of experiments involving Bragg gratings to assess the core uniformity and birefringence [3]. Here, we present the first demonstration of sensing capability in flat fibre, using the principle of shifting the Bragg wavelength. In order to enable a refractive index sensor the optical mode of the Bragg grating must penetrate the analyte, and this is done by removing some of the cladding $[4,5]$.

\section{Theory}

Our direct UV writing arrangement consists of two focused, overlapping UV laser beams which give an inherent interference pattern that is near-circular and of micron order. Exposure of this intensity pattern into the photosensitive core of a flat fiber results in a change in refractive index. If the beam is left on and translated the intra-spot interference pattern is averaged out, resulting in a channel waveguide. More interestingly, by modulating the writing beam during sample translation channel waveguides with integral index-matched planar Bragg gratings can be made [2]. The combination of these two techniques allows planar Bragg gratings to be inserted into complex UV-written devices in a single processing step, and is achievable as a direct result of the small writing spot developed for this process. 


\section{Results}

To allow the evanescent field of the guided mode to be sensitive to the ambient surroundings one side face of the flat fibre was polished back to expose the core layer. The substrate was then hydrogen loaded at a pressure of 120bar for 7days to increase the photosensitivity of the core layer. The light circuit that was direct UV written is depicted in Figure 2 (a), which shows a series of $1 \mathrm{~mm}$ long Bragg gratings stepped closer to the polished edge. The final grating is in close proximity to the edge. Staggering the gratings in such a fashion means the corresponding evanescent fields will have different penetration depths into the analyte that coats it. Using a stepping technique such as this it should be possible to distinguish bulk index changes from those limited to the surface by comparing each of the grating shifts.

(a)

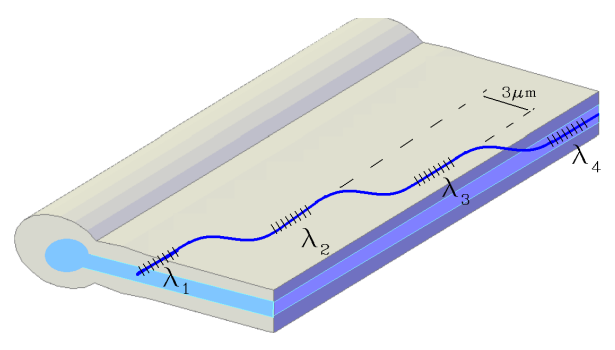

(b)

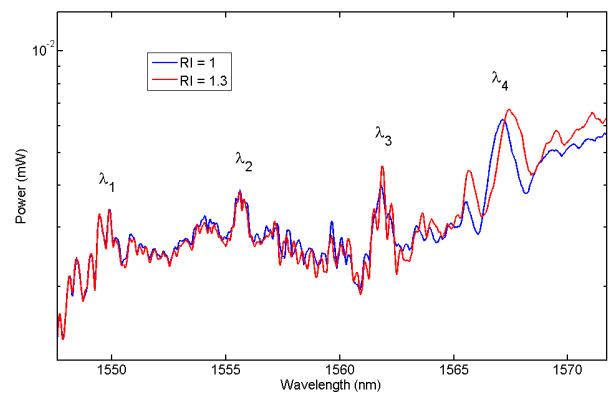

Figure 2: The reflection spectra of four Bragg gratings separated by $3 \mu \mathrm{m}$, leading up to a polished edge.

The preliminary data in Figure 2 (b) shows the observed wavelength shift for a Transverse Magnetic (TM) mode when the sample is exposed to air and a 1.3 refractive index oil. The wavelength shift of the grating closest to the edge is $400 \mathrm{pm}$, which is a 10 -fold increase in sensitivity compared to similiar sensors wrtten in a silica on silicon wafer [6].

It is assumed that the grating furthest from the edge $(9 \mu \mathrm{m})$ is insensitive to the analyte and is thus used as a temperature reference. Typically for this sample of flat fibre a $14 \mathrm{pm}^{o} \mathrm{C}^{-1}$ shift was observed.

\section{Conclusion}

Flat-fiber has been demonstrated as a novel hybrid format that combines the advantages of existing low-cost fiber drawing with the functionality of planar lightwave circuits. Adapted from MCVD fiber fabrication, our preforms are deposited and collapsed into a rectangular geometry before drawing, resulting in extended lengths of mechanically flexible material with a photosensitive germanium-doped planar core.

We shall present the first results of an evanescent field sensor in flat fibre. Discussion shall then turn to flat fibre flexibility, birefringence manipulation and photosensitivity optimisation; all of which will provide additional robustness in the measurements and approach, to new flat fibre applications. Future work shall focus upon stress induced sensing and parallel sensing configurations for transfer to the lab on a chip format and the development of extended lengths of parallel devices for quasi-distributed biochemical and temperature sensing applications.

\section{References}

[1] Webb A. S. Adikan F. R. M. et al. Mcvd planar substrates for uv-written waveguide devices. Electronics Letters, 34 9:517-519, 2007.

[2] G. D. Emmerson S. P. Watts et al. Fabrication of directly uv-written channel waveguides with simultaneously defined integral bragg gratings. Electronics Letters, 38:1531-1532, 2002.

[3] Adkin R.M. et al. First demonstration of direct uv written bragg gratings in collapsed fibre planar samples. European Conference on Optical Communications, Cannes We3.P.69, 2006.

[4] Chryssis A. N. Saini S. et al. Detecting hybridization of dna by highly sensitive evanescent field etched core fiber bragg grating sensors. IEEE Journal on Selected Topics in Quantum Electronics, 11:864-872, 2005.

[5] Iadicicco A. Cusano A. et al. Thinned fiber bragg gratings as refractive index sensors. IIEEE Sensors Journal, 5:1288-1295, 2005.

[6] Gates J.C. Holmes C. et al. New geometry for planar uv written refractive index sensors. SPIE-The International Society for Optical Engineering, 2007. 